daily, one with a bacteriostatic detergent and the other with a shampoo. They tested the comedogenic effect of soaps and shampoos on rabbits' ears and found that seven out of 21 soaps and 10 out of 18 shampoos had some effect, usually slight. They did not produce acne experimentally in man, and they acknowledge that the rabbit's ear may not reflect accurately what happens in patients, but nevertheless they believe on clinical grounds that acne detergicans exists as a rare phenomenon among obsessional washers. They also suggest that washing may play an important part in aggravating acne vulgaris in the less obsessional. In any case, they argue, washing does little to help acne vulgaris and it is not an important part of treatment-an opinion with which not all dermatologists would agree. Be that as it may, their suggestion needs to be taken seriously, since it is clearly undesirable to recommend any form of treatment in acne vulgaris that can give rise to comedones. We should, perhaps, advise washing in acne vulgaris only in moderation and tell our patients to avoid soaps and other detergents that contain halogenated bacteriostatic agents, especially hexachlorophane, which the authors found to be more comedogenic than the other bacteriostatics tested.

${ }^{1}$ Mills, O H, and Kligman, A M, Archives of Dermatology, 1975, 111, 65.

\section{Diagnosis of Down's syndrome at birth}

Down's syndrome may be difficult to diagnose at birth, and normal babies may have some of the features (the term mongolism is now thoroughly outmoded and should be replaced by Down's syndrome ${ }^{1}$ ). In his thesis on newborn infants with the syndrome $\mathrm{Hall}^{2}$ selected ten cardinal features. The most constant of these were flat facial profile, abundant neck skin, dysplastic ears, muscle hypotonia, and radiological evidence of a dysplastic pelvis. These signs are usual in affected babies and unusual in normal newborns, and they are relatively easy to assess. Other typical features are a round head, protruding tongue, iris spots, blunt inner eye angle, and a short broad hand and incurving little finger with a short middle phalanx. The skin is pale, the cry weak, the Moro reflex depressed, and characteristically spontaneous movement is reduced. Some features are less prominent at birth than in infancy - the epicanthic folds, high palate, plantar furrow, and widely spaced first and second toes. Congenital heart lesions are present in about $40 \%$ of children with Down's syndrome. Most have atrial or ventricular septal defects or endocardial cushion defects. Affected infants born preterm present a greater diagnostic problem, because features such as hypotonia, loose pale skin, and the rounded head are absent.

Selective antenatal screening by chromosome analysis of amniotic fluid cells should greatly reduce the numbers of infants born with Down's syndrome. Nevertheless, meanwhile, despite the advances made in cytogenetics since the first demonstration of trisomy 21 by Lejeune et $a l^{3}$ in 1959, clinical diagnosis needs to be improved, especially in the newborn. Paediatricians and general-practitioner obstetricians require, firstly, simple criteria for the decision to request chromosome analysis, especially when only one or two features are present; and, secondly, a reasonably confident clinical diagnosis while awaiting the results. This is most important in the early supportive management of the parents of a child with Down's syndrome, who need to be told the diagnosis as soon as the physician is convinced of its reliability. ${ }^{4}$ The particular problem of duodenal atresia in a child suspected of having Down's syndrome is best managed at a centre where bone marrow aspiration for chromosome examination of mitotic cells can give a rapid diagnosis ${ }^{5}$ and a balanced paediatric surgical decision be made.

Dermatoglyphic analysis is objective and probably more accurate than any list of loosely defined physical features. Reed et $a l^{6}$ devised a diagnostic nomogram based on the palmar triradius. Unfortunately these palm prints and ridge patterns are difficult to obtain in newborns and are therefore not ideal for general use. With practice, triradii and ridge patterns can usefully be observed with an auroscope, but accuracy in the use of a nomogram depends on angle measurement and ridge counts from prints.

Recently Jackson et $a l^{7}$ formulated a check list of the ten most discriminating signs in cases of suspected Down's syndrome under the age of 2 years. With computer analysis weighted correlation coefficients were given to the signs, and summation allowed a positive diagnosis if the cut-off figure was exceeded. The accuracy claimed-93\%-compares well with dermatoglyphic methods. Nevertheless, the list still included some signs which are open to wide subjective interpretation, such as flat nasal bridge, incurved fifth finger, and short neck, and others which are not very prominent in the newborn, such as narrow palate, nystagmus, and separation of the first and second toes. A check list more appropriate to newborns is needed. If one could be devised with similar accuracy and reproducible in normal practice an important advance would be made in clinical diagnosis.

${ }^{1}$ Allen, G, et al, Lancet, 1961, 1, 775.

${ }^{2}$ Hall, B, Acta Paediatrica Scandinavica, 1964, suppl 154.

${ }^{3}$ Lejeune, J, Gauthier, M, and Turpin, R, Comptes Rendus de l'Académie des Sciences, 1959, 248, 602

- Oster, J, and Van den Tempel, A, Acta Paediatrica Scandinavica, 1975, 64, 505 .

5 Smithies, A, and Valman, H B, Lancet, 1974, 1, 1056

${ }^{6}$ Reed, T E, et al, fournal of Pediatrics, 1970, 77, 1024

' Jackson, J F, North, E R, and Thomas, J G, Clinical Genetics, 1976, 9, 483.

\section{Latent schistosomiasis}

Tropical diseases may sometimes stay latent for months and even years after a traveller's return to his temperate homeland. Their presentation may then be perplexing, and a timely reminder has come from the USA of the many ways in which infection with schistosomiasis can become manifest. Both aspects of the problem were presented in the single case of a man who developed paraplegia and skin lesions some seven years after his last possible exposure to infection, the parasite being Schistosoma mansoni.

The longevity of the schistosomal worm in the human host is well documented ${ }^{2}$ and may certainly be over 20 years. What is less well known, however, is that during these 20 years the patient may be substantially free of symptoms and physical signs. After the end of a prolonged latent period neurological complications may develop-both paraplegia and deposits of eggs in the brain (especially with $S$ japonicum) which may mimic a cerebral tumour; while in the case of $S$ mansoni and $S$ japonicum prolonged and symptomless egg 